\title{
The geometric origin of Lie point symmetries of the Schrödinger and the Klein Gordon equations
}

\author{
ANDRONIKOS PALIATHANASIS \\ Faculty of Physics, Department of Astrophysics - Astronomy - Mechanics, University of Athens, \\ Panepistemiopolis, Athens 157 83, Greece \\ anpaliat@phys.uoa.gr \\ MICHAEL TSAMPARLIS \\ Faculty of Physics, Department of Astrophysics - Astronomy - Mechanics, University of Athens, \\ Panepistemiopolis, Athens 157 83, Greece \\ mtsampa@phys.uoa.gr
}

\begin{abstract}
We determine the Lie point symmetries of the Schrödinger and the Klein Gordon equations in a general Riemannian space. It is shown that these symmetries are related with the homothetic and the conformal algebra of the metric of the space respectively. We consider the kinematic metric defined by the classical Lagrangian and show how the Lie point symmetries of the Schrödinger equation and the Klein Gordon equation are related with the Noether point symmetries of this Lagrangian. The general results are applied to two practical problems a. The classification of all two and three dimensional potentials in a Euclidian space for which the Schrödinger equation and the Klein Gordon equation admit Lie point symmetries and $b$. The application of Lie point symmetries of the Klein Gordon equation in the exterior Schwarzschild spacetime and the determination of the metric by means of conformally related Lagrangians.
\end{abstract}

Keywords: Lie symmetries; Noether symmetries; Schrödinger equation; Klein Gordon equation

\section{Introduction}

A systematic method to facilitate the solution of differential equations (DE) is the use of Lie symmetries, because the latter provide the first order invariants which can be used to reduce the DE. The Schrödinger equation and the Klein Gordon equation are two important equations of Quantum Physics. Therefore it is important that we determine their Lie point symmetries and use them either in order to find invariant solutions using Lie symmetry methods [12] or to obtain first integrals which will ease the search for analytic solutions.

Nowadays the determination of Lie symmetries of DEs can be done mechanically by symbolic computer programs, for instance CRACK, MATHLIE, DIMSYM, SADE and others (see [34/5I6] and references therein). However this option is prohibited when one works in Riemannian spaces and in higher dimensions. Therefore it is important that one finds geometric arguments which provide the Lie symme- 
A. Paliathanasis, M. Tsamparlis

tries of DEs in general Riemannian spaces irrespectively of the (finite) dimension $f$ the space. Indeed one finds in the recent literature ([7/8/9|10|11|1213]) which provide the Lie symmetry vectors directly from the collineations of the metric.

The Lie symmetries of the heat equation and the Poisson equation in a general Riemannian space have been determined in previous works [1213. We use these results to find the Lie symmetries of Schrödinger and the Klein Gordon equation in a general Riemannian space.

An important element of the present study is the concept of conformally related Lagrangians, that is Lagrangians which under a conformal transformation of the metric and the potential lead to the same equations but for different dynamic variables. The condition for this is that the Hamiltonian vanishes. Because the dynamic variables of these Lagrangians are not the standard ones in general the Hamiltonian is not relevant to the energy of the system.

From each Lagrangian describing a dynamical system we define a metric, called the kinematic metric, characteristic to the dynamical system described by this Lagrangian. As it will be shown the conformal symmetries of this metric are in close relation with the Noether point symmetries of the equations of motion. Furthermore the kinetic metric of the Lagrangian defines the Laplace operator, hence the corresponding Klein Gordon equation whose Lie symmetries are consequently expressed in terms of the conformal symmetries of the kinematic metric.

The structure of the paper is as follows. In section 2 we give a brief account of the basic properties of Lie point symmetries of differential equations and the conformal algebra of a Riemannian space. In section 3 we consider the classical Lagrangian

$$
L\left(x^{k}, \dot{x}^{k}\right)=T\left(x^{k}, \dot{x}^{k}\right)-V\left(x^{k}\right), \dot{x}^{k}=\frac{d x^{k}}{d t}
$$

of a particle moving in a Riemannian space under the action of the potential $V\left(x^{k}\right)$, where $T\left(x^{k}, \dot{x}^{k}\right)$ is the Kinetic energy defined as follows $T=\frac{1}{2} g_{i j}\left(x^{k}\right) \dot{x}^{i} \dot{x}^{j}$ and $g_{i j}$ is the metric of the Riemannian space. We recall some basic results concerning the relation of Noether point symmetries of the Lagrangian with the homothetic algebra of the metric $g_{i j}$. Then we show that the Noether symmetries of two conformally related Lagrangians are generated from the common conformal algebra.

In section 4 we study the Lie point symmetries of the Schrödinger and the Klein Gordon equations in a general Riemannian space and show that the Lie symmetries are generated from the conformal algebra of the metric which defines the Laplace operator. Using the geometric character of Noether symmetries for the classical Lagrangian and that of the Lie point symmetries of the Schrödinger and of the Klein Gordon equation we establish the connection between the two. More specifically it is shown that if an element of the homothetic algebra of the kinetic metric generates a Noether point symmetry for the classical Lagrangian then it also generates a Lie point symmetry for the Schrödinger equation.

In section 5 we consider the case of Noether point symmetries of the classical 
Lagrangian which are generated from a gradient Killing vector (KV) or a gradient homothetic vector (HV) and show that the Lie symmetry in both cases is a non-local symmetry of the Klein Gordon equation.

In sections 6 and 7 we demonstrate the use of the previous general results to various interesting practical situations. In particular in section [ 6 we find all two and three dimensional potentials in a Euclidian space for which the Schrödinger and the Klein Gordon equations admit non trivial Lie point symmetries. In section 7 we apply the results of section 4 in order to derive invariant solutions of the Wheeler-DeWitt equation and solve the field equations by means of conformally related Lagrangians in a static spherical symmetric spacetime. Finally in section 8 we close with the conclusion.

\section{Symmetries of DEs and collineations of Riemannian spaces}

For the convenience of the reader and the completeness of the paper we state briefly some basic results concerning the Lie point symmetries of differential equations and the conformal algebra of a Riemannian space.

\subsection{Lie symmetries of DEs}

A partial differential equation (PDE) is a function $H=H\left(x^{i}, u, u_{, i}, u_{, i j}, ..\right)$ in the jet space $\bar{B}_{\bar{M}}$, where $x^{i}$ are the independent variables and $u^{A}$ are the dependent variables. The infinitesimal transformation

$$
\begin{aligned}
\bar{x}^{i} & =x^{i}+\varepsilon \xi^{i}\left(x^{k}, u\right) \\
\bar{u} & =\bar{u}+\varepsilon \eta\left(x^{k}, u\right)
\end{aligned}
$$

where $\varepsilon$ is an infinitesimal parameter, is a Lie point symmetry of $H$ with generator

$$
X=\xi^{i}\left(x^{k}, u^{B}\right) \partial_{t}+\eta^{A}\left(x^{k}, u^{B}\right) \partial_{B}
$$

if there exist a function $\lambda$ such that the following condition holds [12]

$$
X^{[n]} H\left(x^{i}, u, u, i, u, i j, . .\right)=\lambda H\left(x^{i}, u, u, i, u, i j, . .\right), \bmod H=0 .
$$

$X^{[n]}$ is the nth prolongation of (3) defined as follows

$$
X^{[n]}=X+\eta_{[i]} \partial_{u_{i}}+\ldots+\eta_{\left[i j \ldots i_{n}\right]} \partial_{u_{i j \ldots i n}}
$$

where

$$
\begin{gathered}
\eta_{[i]}=D_{i} \eta-u_{, j} D_{i} \xi^{j} \\
\eta_{\left[i j . i_{n}\right]}=D_{i_{n}} \eta_{\left[i j . . i_{n-1}\right]}-u_{i j . . k} D_{i_{n}} \xi^{k} .
\end{gathered}
$$

The Lie point symmetry vector field $\mathbf{X}=\xi\left(t, x^{k}\right) \partial_{t}+\eta^{i}\left(t, x^{k}\right) \partial_{x^{i}}$ of the field equations resulting from the Lagrangian function $L=L\left(t, x^{k}, \dot{x}^{k}\right)$, is a Noether 
A. Paliathanasis, M. Tsamparlis

point symmetry of these equations if there exist a function $f=f\left(t, x^{k}\right)$ so that the following condition holds [1]

$$
\mathbf{X}^{[1]} L+\frac{d \xi}{d t} L=\frac{d f}{d t}
$$

$\mathbf{X}^{[1]}$ is the first prolongation of $\mathbf{X}$. The corresponding Noether integral is

$$
I=\xi\left(\frac{\partial L}{\partial \dot{x}^{k}} \dot{x}^{k}-L\right)-\eta^{i} \frac{\partial L}{\partial x^{i}}+f
$$

\subsection{Collineations of Riemannian spaces}

In the following $L_{\xi}$ denotes Lie derivative with respect to the vector field $\xi^{i}$. A vector field $\xi^{i}$ is a CKV of a metric $g_{i j}$ if

$$
L_{\xi} g_{i j}=2 \psi g_{i j}
$$

where $L_{\xi}$ denotes Lie derivative with respect to the vector field $\xi^{i}$. If $\psi=0$ then $\xi^{i}$ is a Killing vector $(\mathrm{KV})$, if $\psi_{, i}=0$ then $\xi^{i}$ is a homothetic vector (HV) and if $\psi_{; i j}=0, \xi^{i}$ is a special conformal Killing vector (sp.CKV). If a CKV is a not a $\mathrm{KV} / \mathrm{HV}$ or sp.CKV is called a proper CKV [14].

Two metrics $g_{i j}, \bar{g}_{i j}$ are conformally related if $\bar{g}_{i j}=N^{2} g_{i j}$ where the function $N^{2}$ is the conformal factor. If $\xi^{i}$ is a CKV of the metric $\bar{g}_{i j}$ so that $L_{\xi} \bar{g}_{i j}=2 \bar{\psi} \bar{g}_{i j}$ then $\xi^{i}$ it is also a CKV of the metric $g_{i j}$, that is, $L_{\xi} g_{i j}=2 \psi g_{i j}$ where the conformal factor

$$
\psi=\bar{\psi} N^{2}-N N_{, i} \xi^{i}
$$

The last relation implies that two conformally related metrics have the same conformal algebra but with Killing/homothetic/special conformal subalgebras spanned by different vector fields. For example a KV for one may be proper CKV for the other. This is an important observation which shall be useful in the following sections.

\section{Conformal Lagrangians and Noether symmetries}

In this section we examine the construction of Noether point symmetries of the classical Lagrangian and the transition of these symmetries to conformally related Lagrangians.

Consider the Lagrangian of a particle moving under the action of a potential $V\left(x^{k}\right)$ in a Riemannian space with metric $g_{i j}$

$$
L=\frac{1}{2} g_{i j} \dot{x}^{i} \dot{x}^{j}-V\left(x^{k}\right)
$$

where $\dot{x}=\frac{d x}{d t}$. The equations of motion follow from the action

$$
S=\int d t\left(L\left(x^{k}, \dot{x}^{k}\right)\right)=\int d t\left(\frac{1}{2} g_{i j} \dot{x}^{i} \dot{x}^{j}-V\left(x^{k}\right)\right) .
$$


Consider the change of variable $t \rightarrow \tau$ defined by the requirement

$$
d \tau=N^{2}\left(x^{i}\right) d t
$$

In the new coordinates $\left(\tau, x^{i}\right)$, the action becomes

$$
S=\int \frac{d \tau}{N^{2}\left(x^{k}\right)}\left(\frac{1}{2} g_{i j} N^{4}\left(x^{k}\right) x^{i} x^{\prime j}-V\left(x^{k}\right)\right)
$$

where $x^{i}=\frac{d x}{d \tau}$ and the Lagrangian is transformed to the new Lagrangian

$$
\bar{L}\left(x^{k}, x^{\prime k}\right)=\frac{1}{2} N^{2}\left(x^{k}\right) g_{i j} x^{\prime i} x^{\prime j}-\frac{V\left(x^{k}\right)}{N^{2}\left(x^{k}\right)} .
$$

If we consider a conformal transformation (not a coordinate transformation!) of the metric $\bar{g}_{i j}=N^{2}\left(x^{k}\right) g_{i j}$ and a new potential function $\bar{V}\left(x^{k}\right)=\frac{V\left(x^{k}\right)}{N^{2}\left(x^{k}\right)}$, then, the new Lagrangian $\bar{L}\left(x^{k}, x^{k}\right)$ in the new coordinates $\tau, x^{k}$ takes the following form,

$$
\bar{L}\left(x^{k}, x^{\prime k}\right)=\frac{1}{2} \bar{g}_{i j} x^{i} x^{\prime j}-\bar{V}\left(x^{k}\right)
$$

which is of the same form as the Lagrangian $L$ in equation (7). Lagrangians $L\left(x^{k}, \dot{x}^{k}\right)$ and $\bar{L}\left(x^{k}, x^{\prime k}\right)$ will be called conformal. In this framework, the action remains the same, i.e. it is invariant under the change of parameter and the equations of motion in the new variables $\left(\tau, x^{i}\right)$ will be the same with the equations of motion for the Lagrangian $L$ in the original coordinates $\left(t, x^{k}\right)$.

In [11, it was shown that the Noether symmetries of a Lagrangian of the form (7) follow from the homothetic algebra of the metric $g_{i j}$. The same applies to the Lagrangian $\bar{L}\left(x^{k}, x^{\prime k}\right)$ and the metric $\bar{g}_{i j}$. The conformal algebra of the metrics $g_{i j}, \bar{g}_{i j}$ are (as a set) the same but their closed subgroups of HVs and KVs are spanned (in general) by different vector fields [15]. This observation leads us to the conclusion, that the Noether point symmetries of the conformally related Lagrangians $L\left(x^{k}, \dot{x}^{k}\right)$ and $\bar{L}\left(x^{k}, x^{k}\right)$ are contained in the common conformal algebra of the metrics $g_{i j}, \bar{g}_{i j}$ [16.

In the following section using the general results of [13] we study the Lie point symmetries of Schrödinger and of the Klein Gordon equation in Riemannian manifolds.

\section{Lie symmetries of Schrödinger and the Klein Gordon equation}

Consider the Schrödinger equation 国 (linear diffusion equation)

$$
\Delta u-u, t=V\left(x^{i}\right) u
$$

and the Klein Gordon equation

$$
\Delta u=V\left(x^{i}\right) u
$$

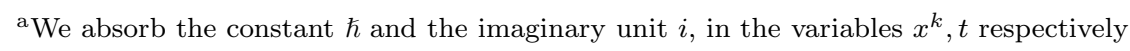


A. Paliathanasis, M. Tsamparlis

in a Riemannian space, where $\Delta$ is the Laplace operator of the Riemannian space with metric $g_{i j}\left(x^{k}\right)$ defined as follows

$$
\Delta u=\frac{1}{\sqrt{|g|}} \frac{\partial}{\partial x^{i}}\left(\sqrt{|g|} g^{i j} \frac{\partial}{\partial x^{j}}\right) u=g^{i j} u_{, i j}-\Gamma^{i} u_{, i} .
$$

$\Gamma_{j k}^{i}\left(x^{k}\right)$ are the Christofell symbols of the metric $g_{i j}$ and $\Gamma^{i}=g^{j k} \Gamma_{j k}^{i}$. In [13] it has been shown that the Lie point symmetries of the second order PDE

$$
A^{a b}\left(x^{c}\right) u_{, a b}-F\left(x^{c}, u, u, c\right)=0
$$

are generated from the elements of the conformal algebra of the second rank tensor $A^{a b}\left(x^{c}\right)$. Equations (13) and (14) are special cases of (16), that is, the Lie point symmetries of the Schrödinger equation and those of the Klein Gordon equation are generated from the conformal algebra of the metric $g_{i j}$. Therefore by applying the general results of [13] we have the following propositions which relate the Lie point symmetries of equations (13) and (14) with the geometry of the underlying Riemannian space which defines the Laplace operator.

Proposition 1 (Lie Symmetries of Schrödinger equation). The Lie point symmetries of Schrödinger equation (13) are generated from the elements of the homothetic algebra of the metric $g_{i j}$ as follows.

a) $Y^{i}$ is a non-gradient $H V / K V$. The generic Lie symmetry vector is

$$
X=\left(2 c \psi t+c_{1}\right) \partial_{t}+c Y^{i} \partial_{i}+\left(a_{0} u+b(t, x)\right) \partial_{u}
$$

with constraint equation

$$
H(b)-b V=0, \quad c L_{Y} V+2 \psi c V+a_{0}=0 .
$$

where $b\left(t, x^{k}\right)$ satisfies (13).

b) $Y^{i}=H^{, i}$ is a gradient $H V / K V$. The generic Lie symmetry vector is

$$
X=\left(2 \psi \int T d t\right) \partial_{t}+T S^{, i} \partial_{i}+\left(\left(-\frac{1}{2} T, t S+F(t)\right) u\right) \partial_{u}
$$

with constraint equation

$$
L_{H} V+2 \psi V-\frac{1}{2} c^{2} H+d=0
$$

and the functions T, F satisfies the following system

$$
T_{, t t}=c^{2} T, \frac{1}{2} T_{, t} \psi+F_{, t}=T d .
$$

Similarly for the Klein Gordon equation (14) we have the following result

Proposition 2 (Lie symmetries of Klein Gordon equation). The Lie point symmetries of Klein Gordon equation (14) are generated from the CKVs of the metric $g_{i j}$, defining the Laplace operator, as follows

a) for $n>2$, the generic Lie symmetry vector is

$$
X=\xi^{i}\left(x^{k}\right) \partial_{i}+\left(\frac{2-n}{2} \psi\left(x^{k}\right) u+a_{0} u+b\left(x^{k}\right)\right) \partial_{u}
$$


where $\xi^{i}$ is a $C K V$ with conformal factor $\psi\left(x^{k}\right), b\left(x^{k}\right)$ is a solution of 114) and the following condition is satisfied

$$
\xi^{k} V_{, k}+2 \psi V-\frac{2-n}{2} \Delta \psi=0 .
$$

b) for $n=2$, the generic Lie symmetry vector is

$$
X=\xi^{i}\left(x^{k}\right) \partial_{i}+\left(a_{0} u+b\left(x^{k}\right)\right) \partial_{u}
$$

where $\xi^{i}$ is a $C K V$ with conformal factor $\psi\left(x^{k}\right), b\left(x^{k}\right)$ is a solution of 14) and the following condition is satisfied

$$
\xi^{k} V_{, k}+2 \psi V=0
$$

Let the potential $V\left(x^{k}\right)$ be

$$
V\left(x^{k}\right)=-\frac{n-2}{4(n-1)} R\left(x^{k}\right)+\bar{V}\left(x^{k}\right)
$$

where $R\left(x^{k}\right)$ is the Ricci scalar of the metric $g_{i j}$. In that case, equation (14) is called the conformal Klein Gordon equation (or Yamabe equation). The conformal Klein Gordon equation plays a central role in the study of a conformal class of metrics by means of the Yamabe invariant (see e.g. [17]).

Replacing $V\left(x^{k}\right)$ from (25) in the Lie symmetry condition (22), we have

$$
\xi^{k} \bar{V}_{, k}+2 \psi \bar{V}-\frac{n-2}{4(n-1)}\left(\xi^{k} R_{, k}+2 R \psi\right)-\frac{2-n}{2} \Delta \psi=0 .
$$

Because $\xi^{i}$ is a CKV with conformal factor $\psi$ it follows that 22]

$$
\xi^{k} R_{, k}=-2 \psi R-2(n-1) \Delta \psi
$$

and this implies

$$
\left(\frac{2-n}{2} \Delta \psi+\frac{n-2}{4(n-1)} \xi^{k} R_{, k}+\frac{n-2}{2(n-1)} \psi R\right)=0 .
$$

Therefore for the conformal Klein Gordon equation the symmetry condition (22) of theorem 2 takes the final form

$$
\xi^{k} \bar{V}_{, k}+2 \psi \bar{V}=0
$$

In the case where $V\left(x^{k}\right)=0$, the Klein Gordon equation becomes the Laplace equation $\Delta u=0$ and if $V\left(x^{k}\right)=-\frac{n-2}{4(n-1)} R\left(x^{k}\right)$ we have the conformal Laplace equation

$$
\Delta u+\frac{n-2}{4(n-1)} R\left(x^{k}\right) u=0 .
$$

A direct result, which arises from Proposition 2 and the symmetry condition (28) for the Yamabe equation, is that, if $V^{n}$ is an $n(n>2)$ dimensional Riemannian space, then, if the Laplace equation in $V^{n}$ is invariant under a Lie group $G_{L}$, then, $G_{L}$ is a subgroup of $\bar{G}_{L_{C}}$, i.e. $G_{L} \subseteq \bar{G}_{L C}$ where $\bar{G}_{L C}$ is a Lie group which leaves invariant 
A. Paliathanasis, M. Tsamparlis

the conformal Laplace equation. The Lie algebras $G_{L}, \bar{G}_{L C}$ are identical if $V^{n}$ does not admit proper CKVs or if all the conformal factors of the CKVs of $V^{n}$ are solutions of the Laplace equation. Moreover, if $V^{n}$ is a conformally flat spacetime then, the conformal Laplace equation (29) admits a Lie algebra of $\frac{1}{2}(n+1)(n+2)+2$ dimension. For instance, the Laplace equation in the three dimensional sphere $S^{3}$ admits eight Lie point symmetries 23 . (the six KVs of $S^{3}$ plus the trivial symmetries $u \partial_{u}, b\left(x^{k}\right) \partial_{u}$ ) while, on the contrary, the conformal Laplace equation admits twelve Lie symmetries, i.e. the conformal algebra of $S^{3}$ plus the trivial symmetries.

The Lie point symmetries of Schrödinger equation follow from the elements of the homothetic algebra of the metric $g_{i j}$, whereas the Lie point symmetries of the Klein Gordon equation are generated by the CKVs of the metric which defines the Laplace operator. Consequently a relation between the Noether point symmetries of the classical Lagrangian (77) and the Lie point symmetries of equations (13) and (14) is expected.

From the form of the symmetry vectors and the symmetry conditions for the Schrödinger equation (for details see [11]) we have the following result.

Proposition 3. If a $K V / H V$ of the metric $g_{i j}$ produces a Lie point symmetry of the Schrödinger equation (13), then generates a Noether point symmetry for the Lagrangian (7) in the space with metric $g_{i j}$ and potential $V\left(x^{k}\right)$. The reverse is also true.

Furthermore for the Klein Gordon equation we have the following proposition which relates the Lie point symmetries of (14) with the Noether point symmetries of the conformal Lagrangian.

Proposition 4. The Lie point symmetries of the Klein Gordon equation 14) for the metric $g_{i j}$ which defines the Laplace operator are related to the Noether point symmetries of the classical Lagrangian (7) for the same metric and the same potential as follows

a) If a $K V$ or a $H V$ of the metric $g_{i j}$ generates a Lie point symmetry of the Klein Gordon equation (14), then it also produces a Noether point symmetry for the classical Lagrangian with gauge function a constant.

b) If a special $C K V$ or a proper $C K V$ of the metric $g_{i j}$ generates a Lie point symmetry of the Klein Gordon equation (14), then it also generates a Noether point symmetry with gauge function a constant for the conformally related Lagrangian provided there exists a conformal factor $N\left(x^{k}\right)$ such that the CKV becomes a $K V$ or a $H V$ of the conformal related metric $\bar{g}_{i j}$.

\footnotetext{
${ }^{\mathrm{b}}$ The three dimensional sphere is a conformally flat space and it is maximally symmetric.
} 


\section{Symmetries of the Lagrangian with non constant gauge function and the Klein Gordon equation}

In the previous considerations we have shown that the Lie point symmetries of the Klein Gordon equation induce Noether point symmetries for the classical Lagrangian if the gauge function is a constant. In this section we investigate the case when the induced Noether symmetry has a gauge function which is not a constant. As we shall show, in this case the induced Noether symmetry comes from a generalized Lie symmetry of the Klein Gordon equation. In particular we study the case where the gradient $\mathrm{KV}$ and the gradient $\mathrm{HV}$ of the kinetic metric generates Noether point symmetries for the classical Lagrangian.

\subsection{The oscillator potential}

Consider the Lagrangian of a particle moving in a decomposable Riemannian space (i.e. the space admits a gradient KV [18])

$$
L=\frac{1}{2}\left(\dot{x}^{2}+h_{A B}\left(y^{C}\right) \dot{y}^{A} \dot{y}^{B}\right)+\mu^{2} x+F\left(y^{C}\right)
$$

For general functions $h_{A B}\left(y^{C}\right), F\left(y^{C}\right)$, Lagrangian (30) admits the autonomous Noether symmetry $\partial_{t}$ and two Noether symmetries $e^{ \pm \mu t} \partial_{x}$ [19] which are due to the gradient KV $\partial_{x}$.. The corresponding Noether integrals are

$$
I_{ \pm}=e^{ \pm \mu t} \dot{x} \mp \mu e^{ \pm \mu t} x \text {. }
$$

It is straightforward to show that the combined integral $I_{0}=I_{+} I_{-}$is time independent and equals

$$
I_{0}=\dot{x}^{2}-\mu^{2} x^{2}
$$

The Laplace Klein Gordon equation defined by the same "kinetic" metric and potential is

$$
u_{x x}+h^{A B} u_{A} u_{B}-\Gamma^{A} u_{A}-\mu^{2} x^{2} u-F\left(y^{C}\right) u=0 .
$$

This equation does not admit a Lie point symmetry for general $h_{A B}\left(y^{C}\right), F\left(y^{C}\right)$. However it is separable with respect to $x$ in the sense that the solution can be written in the form $u\left(x, y^{A}\right)=w(x) S\left(y^{A}\right)$. This implies that the operator $\hat{I}=D_{x} D_{x}-\mu^{2} x^{2}-I_{0}$ satisfies $\hat{I} u=0$ which means that the Klein Gordon equation (33) possesses a Lie Bäcklund symmetry [20 21] with generating vector $\bar{X}=\left(u_{x x}-\mu^{2} x^{2}\right) \partial_{u}$.

Concerning the conformal Klein Gordon equation

$$
u_{x x}+h^{A B} u_{A} u_{B}-\Gamma^{A} u_{A}+\frac{n-2}{4(n-1)} R u-\mu^{2} x^{2} u-2 \bar{F}\left(y^{C}\right) u=0
$$

because for a KV $\xi^{a}$ we have $L_{\xi} R=0$ [22] hence $R=R\left(y^{C}\right)$, equation (34) is written in the form of the Laplace Klein Gordon equation with $F\left(y^{C}\right)=2 \bar{F}\left(y^{C}\right)-$ $\frac{n-2}{4(n-1)} R\left(y^{C}\right)$ and the previous results apply. 


\subsection{The Ermakov potential}

We assume now that the classical Lagrangian (7) admits as Noether symmetries the $s l(2, R)$ Lie algebra $\left\{\partial_{t}, \frac{1}{\mu} e^{ \pm 2 \mu t} \partial_{t} \pm e^{ \pm 2 \mu t} r \partial_{r}\right\}$. The Lagrangian is

$$
L=\frac{1}{2}\left(\dot{r}^{2}+r^{2} h_{A B}\left(y^{C}\right) \dot{y}^{A} \dot{y}^{B}\right)+\frac{1}{2} \mu^{2} r^{2}-\frac{F\left(y^{C}\right)}{r^{2}} .
$$

The corresponding Noether integrals are the Hamiltonian $h$ and

$$
\begin{aligned}
& I_{+}=\frac{h}{\mu} e^{2 \mu t}-e^{2 \mu s} r \dot{r}+\mu e^{2 \mu t} r^{2} \\
& I_{-}=\frac{h}{\mu} e^{-2 \mu t}+e^{-2 \mu t} r \dot{r}+\mu e^{-2 \mu t} r^{2} .
\end{aligned}
$$

From the Noether integrals (36), (37) and the Hamiltonian $h$ of (35) we construct the time independent first integral $\Phi_{0}=h^{2}-I_{+} I_{-}$which is

$$
\Phi_{0}=r^{4} h_{D B} \dot{y}^{A} \dot{y}^{B}+2 F\left(y^{C}\right) .
$$

This is the well known Ermakov invariant [24]25]. An alternative way to construct the Ermakov invariant (38) is to use dynamical Noether symmetries [26]. Indeed, one can show that the Lagrangian (35) admits the dynamical Noether symmetry $X_{D}=K_{j}^{i} \dot{x}^{j} \partial_{i}$ where $K^{i j}$ is a Killing tensor of the second rank given by $K^{i j}=h^{A B}$.

The Laplace Klein Gordon equation defined by the Lagrangian (35) is

$$
u_{r r}+\frac{1}{r^{2}} h^{A B} u_{A B}+\frac{n-1}{r} u_{r}-\frac{1}{r^{2}} \Gamma^{A} u_{A}+\mu^{2} r^{2}+\frac{2}{r^{2}} F\left(y^{C}\right)=0 .
$$

This equation does not admit a Lie point symmetry. However it is separable in the sense that $u\left(r, y^{C}\right)=w(r) S\left(y^{C}\right)$. Then the operator

$$
\hat{\Phi}=h^{A B} D_{A} D_{B}-\Gamma^{A} D_{A}+2 F\left(y^{C}\right)-\Phi_{0}
$$

satisfies the equation $\hat{\Phi} u=0$ which means that (39) admits the Lie Bäcklund symmetry with generator $\bar{X}=(\hat{\Phi} u) \partial_{u}[2021$.

Concerning the conformal Klein Gordon equation, the Ricci scalar of the metric (35) and the HV satisfy the condition $L_{H} R+2 R=0$ [22] that is $R=\frac{1}{r^{2}} \bar{R}\left(y^{C}\right)$. Then, as in the case of the gradient $\mathrm{KV}$, we absorb the term $\bar{R}\left(y^{C}\right)$ into the potential and we obtain the same results with the Laplace Klein Gordon equation.

We conclude that although in the two cases considered above the Lie point symmetries do not transfer from the classical to the "quantum" level the generalized symmetries do transfer.

\section{Lie symmetry classification of Schrödinger and Klein Gordon equations in Euclidian space}

In 1128 all two and three dimensional potentials have been determined for which the corresponding Newtonian dynamical systems admit Lie and Noether point symmetries. Using these results and propositions $3 \sqrt[4]{4}$ above we determine in this section 
all potentials for which the Schrödinger and Klein Gordon equation in Euclidian $2 \mathrm{~d}$ and $3 \mathrm{~d}$ space admit Lie point symmetries.

The Schrödinger equation in Euclidian space is

$$
\delta^{i j} u_{i j}+V\left(x^{k}\right) u=u_{t} .
$$

From proposition 3, we have that the potentials for which the Schrödinger equation (40) admits Lie point symmetries are the same with the ones admitted by the classical Lagrangian. Therefore, the results of [1128 apply directly and give all potentials for which the Schrödinger equation (40) admits at least one Lie point symmetry.

Concerning the Klein Gordon equation in flat space, that is the equation

$$
\delta^{i j} u_{i j}+V\left(x^{k}\right) u=0
$$

from proposition 4 we infer that equation (41) admits a Lie point symmetry due to a $\mathrm{HV} / \mathrm{KV}$ for the following potentials taken from the corresponding Tables of 1128 .

(a) Two dimensional: Table 13 (with $c=0$ ) and Table 14 (with $c=0$ ) of [11].

(b) Three dimensional: Table 5, Table A1 (with $p=0$ ) and Table A2 (with $p=0$ ) of [28. In Table 1 and Table 2 we give the potentials for the $2 \mathrm{~d}$ and the $3 \mathrm{~d}$ case for which the Klein Gordon equation (41) admits Lie point symmetries.

Table 1. The 2d (flat) Klein Gordon admits Lie symmetries from the homothetic group

\begin{tabular}{cccc}
\hline Lie Symmetry & $\mathbf{V}(x, y)$ & Lie Symmetry & $\mathbf{V}(x, y)$ \\
\hline$\partial_{x}$ & $f(y)$ & $\partial_{x}+b \partial_{y}$ & $f(y-b x)$ \\
$\partial_{y}$ & $f(x)$ & $(a+y) \partial_{x}+(b-x) \partial_{y}$ & $f\left(\frac{1}{2}\left(x^{2}+y^{2}\right)+a y-b x\right)$ \\
$y \partial_{x}-x \partial_{y}$ & $f(r)$ & $(x+a y) \partial_{x}+(y-a x) \partial_{y}$ & $r^{-2} f(\theta-a \ln r)$ \\
$x \partial_{x}+y \partial_{y}$ & $x^{-2} f\left(\frac{y}{x}\right)$ & $(a+x) \partial_{x}+(b+y) \partial_{y}$ & $f\left(\frac{b+x}{a+x}\right)(a+x)^{-2}$ \\
\hline
\end{tabular}

As we have seen in section 4 the Lie point symmetries of the Klein Gordon equation are generated from the conformal group of the space, therefore in addition to the above we have to consider the admitted CKVs for each case.

\subsection{The two dimensional case}

We recall that the conformal algebra of a two dimensional space is infinite dimensional [29] and in coordinates with line element $d s^{2}=2 d w d z$ are given by the vectors $X=F(w) \partial_{w}+G(w) \partial_{w}$ with conformal factor $\psi=\frac{1}{2}\left(F_{, w}+G_{, z}\right)$. In the coordinates $(w, z)$ the $2 \mathrm{~d}$ Klein Gordon equation (41) is

$$
u_{w z}+V(w, z) u=0 .
$$


Table 2. The 3d (flat) Klein Gordon admits Lie symmetries from the homothetic group

\begin{tabular}{cc}
\hline Lie Symmetry & $\mathbf{V}(\mathbf{x}, \mathbf{y}, \mathbf{z})$ \\
\hline$a \partial_{\mu}+b \partial_{\nu}+c \partial_{\sigma}$ & $f\left(x^{\nu}-\frac{b}{a} x^{\mu}, x^{\sigma}-\frac{c}{a} x^{\mu}\right)$ \\
$a \partial_{\mu}+b \partial_{\nu}+c\left(x_{\nu} \partial_{\mu}-x_{\mu} \partial_{\nu}\right)$ & $+f\left(\frac{c}{2} r_{(\mu \nu)}-b x_{\mu}+a x_{\nu}, x_{\sigma}\right)$ \\
$a \partial_{\mu}+b \partial_{\nu}+c\left(x_{\sigma} \partial_{\mu}-x_{\mu} \partial_{\sigma}\right)$ & $+f\left(x_{\nu}-\frac{1}{|c|} \arctan \left(\frac{|c| x_{\mu}}{\left|a+c x_{\sigma}\right|}\right), \frac{1}{2} r_{(\mu \sigma)}-\frac{a}{c} x_{\sigma}\right)$ \\
$a \partial_{\mu}+b\left(x_{\nu} \partial_{\mu}-x_{\mu} \partial_{\nu}\right)+$ & \\
$+c\left(x_{\sigma} \partial_{\mu}-x_{\mu} \partial_{\sigma}\right)$ & $f\left(x_{\mu}^{2}+x_{\nu}^{2}\left(1-\frac{c^{2}}{b^{2}}\right)+\left(\frac{2 a}{b}+\frac{2 c}{b} x_{\sigma}\right) x_{\nu}, x_{\sigma}-\frac{c}{b} x_{\nu}\right)$ \\
$s o(3)$ linear combination & $F\left(R, b \tan \theta \sin \phi+c \cos \phi-a M_{1}\right)$ \\
$a \partial_{\mu}+b \theta_{(\nu \sigma)} \partial_{\theta_{(\nu \sigma)}}+c R \partial_{R}$ & $\frac{1}{r_{(\nu \sigma)}^{2} f\left(\theta_{(\nu \sigma)}-\frac{b}{c} \ln r_{(\nu \sigma)}, \frac{a+c x_{\mu}}{c r_{(\nu \sigma)}}\right)}$ \\
$\left(a \partial_{\mu}+b \partial_{\nu}+c \partial_{\sigma}+l R \partial_{R}\right)$ & $\frac{1}{\left(a+l x_{\mu}\right)^{2}} f\left(\frac{b+l x_{\nu}}{l\left(a+l x_{\mu}\right)}, \frac{c+l x_{\sigma}}{l\left(a+l x_{\mu}\right)}\right)$ \\
\hline
\end{tabular}

The Lie symmetry condition (22) becomes

$$
(F V)_{, w}+(G V)_{, z}=0
$$

from which follows that there are infinite many potentials for which the $2 \mathrm{~d}$ Klein Gordon equation admits Lie point symmetries.

\subsection{The three dimensional case}

The 3d Euclidian space admits the three sp.CKVs

$$
K_{C}^{\mu}=\frac{1}{2}\left(\left(x^{\mu}\right)^{2}-\left(\left(x^{\sigma}\right)^{2}+\left(x^{\nu}\right)^{2}\right)\right) \partial_{i}+x^{\mu} x^{\nu} \partial_{x}+x^{\mu} x^{\nu} \partial_{z}, \mu, \sigma, \nu=1,2,3
$$

with corresponding conformal factor $\psi_{C}=x^{\mu}$.

From the symmetry condition (22) of Proposition 2 follows that a sp.CKV generates the Lie point symmetry $X=K_{C}^{\mu}-\frac{1}{2} x^{\mu} u \partial_{u}$ for the 3 d Klein Gordon (41) only for the potential

$$
V(x, y, z)=\frac{1}{\left(x^{\sigma}\right)^{2}} F\left(\frac{x^{\nu}}{x^{\sigma}}, \frac{\delta_{\kappa \lambda} x^{\kappa} x^{\lambda}}{x^{\sigma}}\right) .
$$

One is possible to continue with the linear combinations of these symmetry vectors and deduce all cases that the $3 \mathrm{~d}$ Klein Gordon equation admits a Lie point symmetry (see [1128]).

These results hold for both the Klein Gordon and the conformal Klein Gordon equation. In particular it can be shown that the results remain valid for the conformal Klein Gordon equation provided the metric defining the conformal Laplacian is conformally flat. 


\section{Spherically symmetric space-time}

In this section we consider the Lie point symmetries of the Klein Gordon equation in a non-flat space and in particular in the static spherically symmetric empty space-time, that is the exterior Schwarzschild solution, given by the metric ( $\tau$ is the radial coordinate)

$$
d s^{2}=-a^{2}(\tau) d t^{2}+d \tau^{2}+b^{2}(\tau)\left(d \theta^{2}+\sin ^{2} \theta d \phi^{2}\right) .
$$

The Lagrangian of Einstein field equations for this space-time is [30]31]

$$
L=2 a b^{\prime 2}+4 b a^{\prime} b^{\prime}+2 a
$$

where "'" means derivative with respect to the radius $\tau$. If we see the Lagrangian (44) as a dynamical system in the space of variables $\{a, b\}$, then this system is "autonomous" hence admits the Noether point symmetry $\partial_{\tau}$ with corresponding Noether integra] the "Hamiltonian" $h=$ constant. We compute

$$
h=2 a b^{2}+4 b a^{\prime} b^{\prime}-2 a .
$$

It is straightforward to show that $h=\frac{2}{a} G_{1}^{1}$ where $G_{1}^{1}$ is the Einstein tensor. Because the space is empty from Einstein's equations follows that $h=0$. EulerLagrange equations are

$$
\begin{gathered}
a^{\prime \prime}-\frac{a}{2 b^{2}} b^{2}+\frac{1}{b} a^{\prime} b^{\prime}+\frac{1}{2} \frac{a}{b^{2}}=0 \\
b^{\prime \prime}+\frac{1}{2 b} b^{\prime 2}-\frac{1}{2 b}=0 .
\end{gathered}
$$

We end up with a system of three equations whose solution will give the functions $a(\tau), b(\tau)$. However it is found that the solution of the system does not give these functions in the well known closed form. This is due to the Lagrangian we have considered. Indeed as we shall show below it is possible to find the solution in closed form by considering a Lagrangian conformally related to the Lagrangian (44). It is straightforward to show that the Lagrangian (44) admits the Noether symmetry

$$
X_{1}=2 \tau \partial_{\tau}+H
$$

where $H=-2 a \partial_{a}+2 b \partial_{b}$ is a non-gradient homothetic vector of the two dimensional kinetic metric

$$
d \bar{s}^{2}=2 a d b^{2}+4 b d a d b
$$

defined from the Lagrangian (44). Consider the Klein Gordon equation defined by the metric (45) with potential $V(a, b)=2 a$, that is,

$$
-\frac{a}{4 b^{2}} u_{a a}+\frac{1}{2 b} u_{a b}-\frac{1}{4 b^{2}} u_{a}-2 a u=0 .
$$

\footnotetext{
${ }^{\mathrm{c}} h$ is not the "energy" because the coordinate is the radial distance not the time
} 
Equation (46) admits as Lie point symmetries the vectors 31]

$$
\begin{gathered}
u \partial_{u}, b(a, b) \partial_{u} \\
H=-2 a \partial_{a}+2 b \partial_{b} \quad, \quad X_{2}=\frac{1}{a b} \partial_{a}, X_{3}=\frac{a}{2 b} \partial_{a}-\partial_{b}
\end{gathered}
$$

where the vectors $X_{2}, X_{3}$ are proper CKVs of the two dimensional metric (45).

By applying the Lie invariants it is possible to find solutions of the Klein Gordon equation (46) which are invariant with respect to one of the admitted Lie point symmetries.

For example let us consider the Lie point symmetry $H_{u}=H-2 c u \partial_{u}$. The zero order invariants $H_{u}$ are $w=a b, u=a^{c} \Phi(w)$. Replacing in (46) we find the solution

$$
u(a, b)=a^{c}\left[c_{1} I_{c}^{B}(2 \sqrt{2} a b)+c_{2} K_{c}^{B}(2 \sqrt{2} a b)\right]
$$

where $I^{B}, K^{B}$ are the Bessel modified functions [31. Working similarly for the Lie point symmetry $\mathrm{H}+e \mathrm{X}_{2}-c u \partial_{u}$ we find the solution

$$
u(a, b)=\left(a^{2}-e b^{-1}\right)^{\frac{c}{4}}\left[c_{1} I_{-\frac{c}{2}}\left(2 \sqrt{2 b\left(a^{2} b-e\right)}\right)+c_{2} K_{\frac{c}{2}}^{B}\left(2 \sqrt{2 b\left(a^{2} b-e\right)}\right)\right] .
$$

One can find more solutions using linear combinations of these Lie symmetries.

Following proposition 4 we look for a conformal metric for which one of the CKVs $X_{2}, X_{3}$ becomes a $\mathrm{KV}$ and write the corresponding conformally related Lagrangian which admits this $\mathrm{CKV}$ as a Noether symmetry. We consider first the vector $X_{2}$ and the conformally related metric

$$
d s^{2}=N^{2}\left(4 a d b^{2}+8 b d a d b\right)
$$

where $N(a, b)=g(b) \sqrt{a}$ and $g(b)$ is an arbitrary function of its argument. It is easy to show that the vector $X_{2}$ is a KV of this metric hence a Noether symmetry for the family of conformally related Lagrangians

$$
\bar{L}=N^{2}\left[2 a\left(\frac{d b}{d r}\right)^{2}+4 b\left(\frac{d a}{d r}\right)\left(\frac{d b}{d r}\right)\right]+\frac{2 a}{N^{2}}
$$

where we have considered the coordinate transformation $d \tau=\frac{d r}{N^{2}(a, b)}$. The Noether function for this Noether symmetry is the "Hamiltonian" of the Lagrangian (48). This constant and the two Lagrange equations for the "generalized" coordinates $a, b$ provide a system of differential equations which will give the functions $a(\tau), b(\tau)$.

We consider $g(b)=g_{0}=$ constant, that is from the family of Lagrangians (48) we take the Lagrangian

$$
L=g_{0}^{2}\left[2 a^{2}\left(\frac{d b}{d r}\right)^{2}+4 a b\left(\frac{d a}{d r}\right)\left(\frac{d b}{d r}\right)\right]+\frac{2}{g_{0}^{2}} .
$$


For this Lagrangian the field equations are the "Hamiltonian" of the Lagrangian (49)

$$
a^{2}\left(\frac{d b}{d r}\right)^{2}+2 b^{2}\left(\frac{d a}{d r}\right)\left(\frac{d b}{d r}\right)-V_{0}=0
$$

and the Euler Lagrange equations of (49) with respect to the variables $a, b$

$$
\begin{gathered}
\frac{d^{2} a}{d r^{2}}+\frac{1}{a^{2}}\left(\frac{d a}{d r}\right)^{2}+\frac{2}{b}\left(\frac{d a}{d r}\right)\left(\frac{d b}{d r}\right)=0 \\
\frac{d^{2} b}{d r^{2}}=0
\end{gathered}
$$

where we have set $V_{0}=g_{0}^{-4}$.

The solution of the system of equations (50)-(52) is

$$
b(r)=b_{1} r+b_{2} \quad, \quad a^{2}(r)=\frac{V_{0} r+2 a_{1} b_{1}}{2 b_{1}\left(b_{1} r+b_{2}\right)} .
$$

Under the linear transformation $b_{1} r=b_{1} R-\frac{b_{2}}{b_{1}}$ and if we set $V_{0}=2\left(b_{1}\right)^{2}, a_{1}=$ $-2 m+b_{2}$, we obtain the exterior Schwarzschild solution in the standard coordinates

$$
d s^{2}=-\left(1-\frac{2 m}{R}\right) d t^{2}+\left(1-\frac{2 m}{R}\right)^{-1} d R^{2}+R^{2}\left(d \theta^{2}+\sin ^{2} \theta d \phi^{2}\right)
$$

The choice of the function $g(b)$ is essentially a choice of the coordinate system. Obviously the final solution must always be the exterior Schwarzschild solution. Working similarly we find that $X_{3}$ becomes a KV for the conformal metric (47) if $N_{3}(a, b)=f\left(a^{2} b\right) \sqrt{a}$, and generates a Noether point symmetry for the conformal Lagrangian (48) (with $N_{3}$ in the place of $N$ ) and from this point we continue as above.

\section{Conclusion}

We have determined the Lie point symmetries of the Schrödinger equation and the Klein Gordon equation in a general Riemannian space. It has been shown that these symmetries are related with the homothetic algebra and the conformal algebra of the metric of the space. Furthermore these symmetries have been related to the Noether point symmetries of the classical Lagrangian for which the metric $g_{i j}$ is the kinematic metric. More precisely for the Schrödinger equation it has been shown that if a $\mathrm{KV} / \mathrm{HV}$ of the metric $g_{i j}$ produces a Lie point symmetry for the Schrödinger equation then produces a Noether point symmetry for the classical Lagrangian in the space with metric $g_{i j}$ and potential $V\left(x^{k}\right)$. For the Klein Gordon equation the situation is different. That is the Lie point symmetries of the Klein Gordon are generated from the conformal algebra of the metric $g_{i j}$. The KVs and the HV of this group produce a Noether symmetry of the classical Lagrangian with a constant gauge function, however the proper CKVs produce a Noether symmetry 
of the conformal Lagrangian if there exists a conformal factor $N\left(x^{k}\right)$ such that the CKV becomes a KV/HV of $g_{i j}$.

We have applied these general results to two cases of practical interest. The classification of all potentials in Euclidian 2d and 3d space for which the Schrödinger equation and the Klein Gordon equation admit a Lie point symmetry and finally we have considered the Lie point symmetries of the Klein Gordon equation in the static, spherically symmetric empty spacetime. In the last case we have demonstrated the role of the Lie symmetries and that of the conformal Lagrangians in the determination of the closed form solution of Einstein equations.

The knowledge of Lie point symmetries of Schrödinger equation and the Klein Gordon equation in a general Riemannian space makes possible the determination of solutions of these equations which are invariant under a given Lie point symmetry. In addition the Lie point symmetries are used in Quantum Cosmology [32 33]34]35|36] in order to determine the form of solutions of the Wheeler-DeWitt equation in a given Riemannian space.

\section{References}

[1] H. Stephani, Differential Equations: Their Solutions using Symmetry Cambridge University Press (1989) New York

[2] N.H. Ibragimov, Transformation Groups Applied to Mathematical Physics translated from the Russian Mathematics and Its Applications (Soviet Series) D Reidel (1985) Publishing Co.: Dordrecht

[3] T. Wolf and A. Brand, The Computer Algebra Package CRACK for investigating PDEs, Proceedings of ERCIM, Partial Diffential Equations and Group Theory, Bonn, (1992)

[4] G. Baumann, Symmetry Analysis of Differential Equations with Mathematica, Springer, New York, (2000)

[5] J. Sherring, A.K Head and G.E. Prince, Dimsym and LIE: symmetry determination packages, Math. Comput. Modelling 25 (1997) 153

[6] T. M. Rocha Filio and A. Figueiredo, [SADE] A Maple package for the Symmetry Analysis of Differential Equations, Comput. Phys. Commun. 182 (2011) 467

[7] A.V. Aminova, Projective transformations and symmetries of differential equations, Sb. Math. 186 (1995) 1711

[8] A.V. Aminova, Projective geometry of systems of differential equations: general conceptions, Tensors (N.S.) 62 (2000) 65

[9] G.E. Prince, M. Crampin, Projective differential geometry and geodesic conservation laws in general realtivity. I: Projective actions, Gen. Relativ. Gravit. 16 (1984) 921

[10] A. Aslam and A. Qadir, Noether Symmetries of the Area-Minimizing Lagrangian, J. Applied Mathematics, 2012 (2012) 532690

[11] M. Tsamparlis and A. Paliathanasis, Two-dimensional dynamical systems which admit Lie and Noether symmetries, J. Phys. A: Math. and Theor. 44 (2011) 175202

[12] Y. Bozhkov and I.L. Freire, Special conformal groups of a Riemannian manifold and Lie point symmetries of the nonlinear Poisson equation, J. Differential Equations 249 (2010) 872

[13] A. Paliathanasis and M. Tsamparlis, Lie point symmetries of a general class of PDEs: the heat equation, J. Geom. Phys. 62 (2012) 2443

[14] G.H. Katzin, J. Levine and W.R. Davis, Curvature Collineations: A Fundamental 
Symmetry Property of the Space-Times of General Relativity Defined by the vanishing Lie Derivative of the Riemann Curvature Tensor, J. Math. Phys. 10 (1969) 617

[15] K. Yano, The Theory of Lie Derivatives and Its Applications, North Holland, Amsterdam (1956)

[16] M. Tsamparlis, A. Paliathanasis, S. Basilakos and S. Capozziello, Conformally related metrics and Lagrangians and their physical interpretation in cosmology, Gen. Relativ. Gravit. 45 (2013) 2003

[17] J.M. Lee and T.H. Parker, The Yamabe problem, Bull (NS) Am Math Soc 17 (1987) 37

[18] M. Tsamparlis, D. Nikolopoulos and P.S. Apostolopoulos, Computation of the conformal algebra of $1+3$ decomposable spacetimes, Class. Quantum Grav. 15 (1998) 2909

[19] M. Tsamparlis and A. Paliathanasis, Generalizing the autonomous Kepler-Ermakov system in a Riemannian space, J. Phys. A: Math. and Theor. 45 (2012) 275202

[20] W. Miller Jr, The technique of variable separation for partial differential equations, Lectures Notes in Physics 189 (1983) 184

[21] N. Kamran and R.G. McLenaghan, Separation of variables and symmetry operators for the conformally invariant Klein-Gordon equation on curved spacetime, Lett. Math. Phys. 9 (1985) 65

[22] G.S. Hall and J.D. Steele, Conformal vector fields in general relativity, J. Math. Phys. 32 (1991) 1847

[23] I. L. Freire, On the paper "Symmetry analysis of wave equation on sphere" by H. Azad and M.T. Mustafa, J. Math. Anal. Appl., 367 (2010) 716

[24] H.R. Lewis, Classical and quantum systems with time-dependent harmonic-oscillatortype Hamiltonians, Phys. Rev. Lett. 18 (1967) 510

[25] S. Moyo and P.G.L. Leach, A note on the construction of the Ermakov-Lewis invariant, J. Phys. A: Math. Gen. 35 (2002) 5333

[26] T.M. Kalotas and B.G. Wybourne, Dynamical Noether symmetries, J. Phys A: Math and Gen 15 (1982) 2077

[27] E. Pinney, The nonlinear differential equation $y^{\prime \prime}+p(x) y+c y^{-3}=0$, Proc. Am. Math. Soc. 1 (1950) 681

[28] M. Tsamparlis, A. Paliathanasis and L. Karapathopoulos, Autonomous threedimensional Newtonian systems which admit Lie and Noether point symmetries, J. Phys. A: Math. and Theor. 45 (2012) 275201

[29] A.A. Belavin, A.M. Polyakov and A.B. Zamolodchikov, Infinite conformal symmetry in two-dimensional quantum field theory, Nucl. Phys. B241 (1984) 333

[30] B. Vakili, Quantization of the Schwarzschild black hole: a Noether symmetry approach, Int. J. Theor. Phys. 51 (2012) 133

[31] T. Christodoulakis et al, Lie-point and variational symmetries in minisuperspace Einstein's gravity, (2012) arXiv: 1208.0462

[32] S. Capozziello, R. De Ritis and P. Scudellaro, Noether's symmetries in Quantum Cosmology, IJMPD 3 (1994) 609

[33] T. Christodoulakis, G. Kofinas and G.O. Papadopoulos, Conditional symmetries and phase space reduction towards GCT invariant wave functions, for the class A Bianchi type VI and VII vacuum cosmologies, Phys.Lett. B 514 (2001) 149

[34] B. Vakili, Noether symmetric f (R) quantum cosmology and its classical correlations, Phys. Lett. B 669 (2008) 206

[35] B. Vakili and F. Khazaie, Noether symmetric classical and quantum scalar field cosmology, Class. Quantum Grav. 29 (2012) 035015

[36] S. Capozziello, M. De Laurentis and S.D. Odintsov, Hamiltonian dynamics and 
A. Paliathanasis, M. Tsamparlis

Noether symmetries in extended gravity cosmology, EPJC 72 (2012) 2068 